\title{
HIGH PRECISION TARGET CENTER DETERMINATION FROM A POINT CLOUD
}

\author{
Klemen Kregar, Dejan Grigillo and Dušan Kogoj \\ University of Ljubljana, \\ Faculty of Civil and Geodetic Engineering, \\ Chair of Geodesy, \\ Jamova cesta 2, 1000 Ljubljana, Slovenia \\ klemen.kregar@fgg.uni-lj.si
}

KEY WORDS: terrestrial laser scanner, planar target, target detection, point cloud, calibration, registration

\begin{abstract}
:
Many applications of terrestrial laser scanners (TLS) require the determination of a specific point from a point cloud. In this paper procedure of high precision planar target center acquisition from point cloud is presented. The process is based on an image matching algorithm but before we can deal with raster image to fit a target on it, we need to properly determine the best fitting plane and project points on it. The main emphasis of this paper is in the precision estimation and propagation through the whole procedure which allows us to obtain precision assessment of final results (target center coordinates). Theoretic precision estimations - obtained through the procedure were rather high so we compared them with the empiric precision estimations obtained as standard deviations of results of 60 independently scanned targets. An $\chi^{2}$-test confirmed that theoretic precisions are overestimated. The problem most probably lies in the overestimated precisions of the plane parameters due to vast redundancy of points. However, empirical precisions also confirmed that the proposed procedure can ensure a submillimeter precision level. The algorithm can automatically detect grossly erroneous results to some extent. It can operate when the incidence angles of a laser beam are as high as $80^{\circ}$, which is desirable property if one is going to use planar targets as tie points in scan registration. The proposed algorithm will also contribute to improve TLS calibration procedures.
\end{abstract}

\section{INTRODUCTION}

Terrestrial laser scanning (TLS) technology generally does not allow the measuring of a specific point. However many TLS tasks require the determination of a specific point from the point cloud. For example, point-based registration (Lichti and Skaloud, 2010a), geo-referencing of point cloud, and also different methods of scanner calibration (Lichti, 2010), (Reshetyuk, 2010), (Schulz, 2007). The problem can be solved by scanning geometric objects or contrast shapes that can be recognized from point cloud. For different tasks different precisions are desired, in this paper we focus on the highest achievable precisions - below the millimeter.

Most of TLS software have built-in solutions for target center determination. Each scanner manufacturer prefers its own type of targets. Most instruments automatically detect the target center, but in most cases it does not return the precision of coordinates. Often only a flat rate precision estimation is given in the scanner's technical data.

In the literature some authors report about target center determination. (Chow et al., 2010) apply least square circle fitting in the adjusted plane and in (Chow et al., 2011) intersection of lines that distinct different colors of target is applied. (Vezočnik, 2011) in doctoral thesis determines target center for Leica circular planar target. He is fitting 3D surface into intensity image of scanned points projected onto adjusted plane. The main disadvantage of this approach is instability of targets used. The author reports that target centers are moving within the frame of $1 \mathrm{~mm}$ when target is rotated. (Lichti et al., 2000) used targets from low-cost acrylic reflector with diameter of $10 \mathrm{~cm}$. At distances up to $600 \mathrm{~m}$ they obtained multiple laser returns from the same target with different intensities of responses. The target center was determined as a mean of responses weighted by the intensities.

For our research we designed a planar target that should allow center determination with TLS as well as total station (TPS) with submillimeter precision. We propose algorithm that is different from others', explain its advantages and carry out some test to evaluate its performance.

In the second section we explain how the algorithm works. The plane is fitted by minimizing the distances in the direction of the incidence laser beam and not orthogonal distances as is usual. Points are projected on to the plane using an oblique projection and then rasterized into intensity image. Image matching is applied afterwards and target center is transformed back from the plane into 3D space. Target center precision is also determined throughout the procedure.

In the third section the two methods for results evaluation are given. The fourth section leads us through the steps of the algorithm with key intermediate results. Then the findings of the result evaluation methods are presented.

In the conclusion we try to emphasize the advantages of the proposed method along with the main points where the procedure has to be investigated more thoroughly and improved.

\section{TARGET CENTER DETERMINATION ALGORITHM}

Since the proposed procedure of target center determination is based on image matching, it is essential to provide a reference plane to project the scanned points on it. 
The reference plane is the plane best fitted to the scanned points of target. There are often certain amounts of points in a point cloud that do not belong to the target plane. It is wise to remove such outliers for they may cause severe difficulties in the next step of plane adjustment. The RANSAC procedure is used for the detection of points belonging to the plane (Fischler and Bolles, 1981).

\section{$2.1 \quad$ Reference Plane}

Normally we would use least square method to find the plane that minimizes the perpendicular distances from the points to the plane. In our case we wish to minimize the distances in the direction of the incidence laser beam. It is known that single point precision is lower in the direction of measured distance $\rho$ than in perpendicular directions dependant on measured angles; standard error ellipsoid of single point is elongated in the direction of laser beam (Schulz and Ingensand, 2004). Let the normal vector of the plane be $\vec{n}=\left[\begin{array}{lll}a & b & c\end{array}\right]$ and direction of the incidence laser beam $\vec{v}=\left[\begin{array}{lll}t & u & v\end{array}\right]$. Both vectors have to be unit length $\|\vec{n}\|=1$ and $\|\vec{v}\|=1$. For each point $\vec{p}=\left[\begin{array}{lll}X & Y & Z\end{array}\right]$ we need to find the length of the projection of the point to the line defined by $\vec{v}$. The length of the projection of $\vec{p}$ to the $\vec{n}$ must be divided by $\cos \varphi$, where $\varphi$ is angle between $\vec{n}$ and $\vec{v}$. Since they are both unit vectors, $\cos \varphi=\vec{n} \cdot \vec{v}$. The mathematical model for the best fitting plane is

$$
\frac{a X+b Y+c Z}{a t+b u+c v}-d=0
$$

Equation (1) is linearized to the form $\mathbf{A x}+\mathbf{B} \boldsymbol{\Delta}=\mathbf{f}$.

$\mathbf{x}$ is vector of observations (i.e. cloud points coordinates $\left.\left(X_{i}, Y_{i}, Z_{i}\right), i=1, \ldots, n\right)$ and matrix $\mathbf{A}$ contains the derivatives of eqn. (1) with respect to observations $\mathbf{x}$. The size of $\mathbf{A}$ equals $n \times 3 n$ : 1 row and 3 columns for each point.

$\boldsymbol{\Delta}$ is vector of unknowns (i.e. plane parameters $a, b, c$ and $d$ ) and matrix $\mathbf{B}$ contains the derivatives of eqn. (1) with respect to unknowns $\boldsymbol{\Delta}$. The size of $\mathbf{B}$ equals $n \times 4$ : 1 row for each point and 4 columns for 4 unknowns.

The solution is obtained through the Gauss-Markov model

$$
\boldsymbol{\Delta}=\left(\mathbf{B}^{T}\left(\mathbf{A} \mathbf{A}^{T}\right)^{-1} \mathbf{B}\right)^{-1}\left(\mathbf{B}^{T}\left(\mathbf{A} \mathbf{A}^{T}\right)^{-1} \mathbf{f}\right)
$$

Stochastic properties or precisions of plane parameters are obtained through:

$$
\boldsymbol{\Sigma}_{\Delta \Delta}=\sigma_{0}^{2}\left(\mathbf{B}^{T}\left(\mathbf{A} \mathbf{A}^{T}\right)^{-1} \mathbf{B}\right)^{-1}
$$

More detailed descriptions of adjustment can be found in (Grigillo and Stopar, 2003) or (Kuang, 1996). The results of this step are four parameters of the best fitting plane along with their covariance matrix.

\subsection{Projection of points on to the plane}

For our purpose we wish to carry out an oblique projection of points to the plane in the direction of the incidence laser beam. The reason is again significantly lower precision of measured distances $\rho$ relatively to angles $\theta$ and $\alpha$. Each point has to be translated to the plane in the direction of the incidence laser beam. The length of the translation is equal to the shortest distance between point and plane $\vec{p} \cdot \vec{n}$ divided by $\cos \varphi=\vec{p} \cdot \vec{v}$. The vector of translation for each point is

$$
\vec{t}=\vec{v} \frac{\vec{p} \cdot \vec{n}}{\vec{n} \cdot \vec{v}}
$$

Point $\vec{p}$ projected to the plane becomes $\overrightarrow{p^{\prime}}=\vec{p}+\vec{t}$. The result of this step are points lying in the plane, but they still have all three coordinates.

\subsection{Rasterization of planar points}

To obtain a raster image which will serve for image matching, the points have to be transformed into a $2 \mathrm{D}$ coordinate system. We need to define the two basis vectors spanning our plane. They should both be perpendicular to plane normal vector $\vec{n}$ and one unit long. We can obtain them using the Gramm-Schmidt algorithm. Randomly chosen vector $\vec{r}$ is decomposed in a way that one component is perpendicular to $\vec{n}$ and then normalized. The procedure is for practical purposes carried out as

$$
\overrightarrow{e_{1}}=\frac{\vec{n} \times \vec{r}}{|\vec{n} \times \vec{r}|} \quad \overrightarrow{e_{2}}=\frac{\vec{n} \times \overrightarrow{e_{1}}}{\left|\vec{n} \times \overrightarrow{e_{1}}\right|}
$$

Since the covariance matrix of components of $\vec{n}$ is known and $\vec{r}$ is randomly chosen, we are able to obtain covariance matrices $\Sigma_{e 1}$ and $\Sigma_{e 2}$ of vectors $\overrightarrow{e_{1}}$ and $\overrightarrow{e_{2}}$ components through the error propagation law. Points from a plane in $3 \mathrm{D}$ are transformed into a plane spanned by vectors $\overrightarrow{e_{1}}$ and $\overrightarrow{e_{2}}$ through the equation

$$
\left[\begin{array}{ll}
x & y
\end{array}\right]=\left[\begin{array}{lll}
X & Y & Z
\end{array}\right]\left[\begin{array}{ll}
\mathbf{e}_{\mathbf{1}}^{T} & \mathbf{e}_{\mathbf{2}}^{T}
\end{array}\right]
$$

Points in the plane are than rasterized into grid of point intensities. Many modern computational tools such as Matlab ${ }^{\circledR}$ or Mathematica ${ }^{\circledR}$ have such functions built-in. It is important to set the grid resolution according to the scanning resolution. Pixel values are interpolated from point's intensities. It is not very important which method is used for interpolation. In Matlab ${ }^{\circledR}$ we can choose between linear, cubic, natural and the nearest neighbour solutions, but in our research the selected method did not affect final results.

\subsection{Image matching}

Image matching is well established procedure in the field of image processing and digital photogrammetry. In our procedure we are matching two images, the raster image of the target and the template image. Template was generated manually in order to describe actual targets. Pixel values in the template are zeros and ones describing black and white intensities respectively. The procedure consists of two steps. In the first step the correlation coefficients between template and target image are calculated for each possible position of the template in the target image. The approximate (pixel) position of the target center $(\xi, \eta)$ is determined by the greatest value of the correlation coefficient. Procedure is in details described in (Kraus, 2000). In the second step we obtain subpixel position of target center through least squares adjustment (Höhle, 1997).

The final results of image matching are plane coordinates of target center $\left(x_{c}, y_{c}\right)$ along with their covariance matrix $\Sigma_{x y}$. 
2.5 Transformation of the target center from the plane back to space

The obtained coordinates of the target center in the projection plane has to be transformed back to 3D space of the original point cloud. The transformation is the reverse of that in equation (6)

$$
\left[\begin{array}{lll}
X & Y & Z
\end{array}\right]=\left[\begin{array}{ll}
x & y
\end{array}\right]\left[\begin{array}{ll}
\mathbf{e}_{1} & \mathbf{e}_{2}
\end{array}\right]^{T}
$$

The covariances of the obtained point depend on the covariance matrices of the input parameters. Through the error propagation law precisions of the target center are derived as

$$
\Sigma_{X Y Z}=\mathbf{J}\left[\begin{array}{ccc}
\boldsymbol{\Sigma}_{\mathbf{x y}} & 0 & 0 \\
0 & \boldsymbol{\Sigma}_{\mathbf{e} \mathbf{1}} & 0 \\
0 & 0 & \boldsymbol{\Sigma}_{\mathbf{e} 2}
\end{array}\right] \mathbf{J}^{T}
$$

where $\mathbf{J}$ is Jacobian of the equations (7)

$$
J=\left[\begin{array}{llll}
\mathbf{e}_{\mathbf{1}}^{T} & \mathbf{e}_{\mathbf{2}}{ }^{T} & x \mathbf{I}_{3 \times 3} & y \mathbf{I}_{3 \times 3}
\end{array}\right]
$$

Projection from the plane back to the point cloud space can be enhanced by adding $z=0$ coordinate to the plane coordinates $(x, y)$ and expanding the projection matrix $\left[\begin{array}{ll}\mathbf{e}_{\mathbf{1}} & \mathbf{e}_{\mathbf{2}}\end{array}\right]^{T}$ with the plane normal vector $\mathbf{n}$. The covariance matrix of the plane from section 2.1 are added to the covariance matrix of the input parameters from equation (8). The variances of the plane parameters $a, b$ and $c$ refer to the normal $\mathbf{n}$ and the variance of the parameter $d$ refers to the "plane" coordinate $z=0$.

\section{EVALUATION OF RESULTS}

When the target cannot be scanned entirely due to obstacles or data artefacts (Lichti and Skaloud, 2010b) it is impossible to fit the proper plane into a point cloud, causing the results of target determination algorithm to be erroneous. The results of the multiple target center determination will be visually classified as correct or incorrect. Furthermore, the correctness of the result will be presented with respect to the incidence angle and maximal correlation from image matching. We would like to check if correctness can be predicted regarding these two parameters.

Precisions obtained through the proposed procedures turned out to be rather high. Precisions obtained from image matching are always better than the size of a pixel (in our case a millimeter). Imprecisions of the vector base $\left(\mathbf{e}_{1}, \mathbf{e}_{2}, \mathbf{n},\right)$ does not reduce them much. Simple test was set up to validate/justify the achieved precisions.

Five targets were scanned 60 times independently from the same scanner station at a distance of $5 \mathrm{~m}$ and another two targets at a distance of $45 \mathrm{~m}$. The targets were facing different directions so that each target had a different incidence angle of the laser beam: $2^{\circ}, 15^{\circ}, 35^{\circ}, 50^{\circ}$ and $85^{\circ}$ (see Figure 1) for $5 \mathrm{~m}$ distance and $10^{\circ}$ and $65^{\circ}$ for $45 \mathrm{~m}$ distance.

All scans were processed and each target center was determined 60 times, along with its precision, yielding sets $\left(X_{i j}, Y_{i j}, Z_{i j}, \sigma_{X_{i j}}, \sigma_{Y_{i j}}, \sigma_{Z_{i j}}\right)$; where $i$ indicates one of the targets $i=1, \ldots, 7$ and $j$ indicates the iteration $j=1, \ldots, m=$

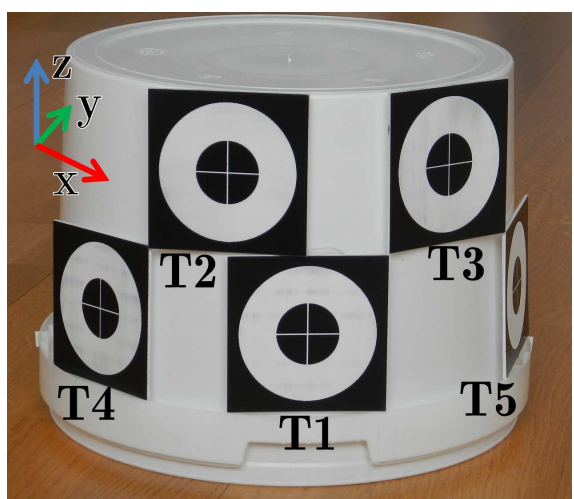

Figure 1: Targets setup

60. For each of the five targets the mean precision was obtained as

$$
\bar{\sigma}_{X_{i}}=\sqrt{\frac{\sum_{j=1}^{m} \sigma_{X_{i j}}^{2}}{m-1}}
$$

On the other hand, the standard deviation of the center coordinates was obtained as

$$
\hat{\sigma}_{X_{i}}=\sqrt{\frac{\sum_{j=1}^{m}\left(X_{i j}-\bar{X}_{i}\right)^{2}}{m-1}}
$$

Precisions of coordinates $Y$ and $Z$ are also calculated through equations (10) and (11)

Statistical testing is carried out to check if theoretical precisions obtained through the process are adequate to empirical precisions i.e. deviations of target centers. An $\chi^{2}$ test is used to check the consistence of variances $\bar{\sigma}$ and $\hat{\sigma}$. Under the assumption of a normal distribution of errors, the test statistic

$$
H=\nu \frac{\bar{\sigma}^{2}}{\hat{\sigma}^{2}}
$$

is distributed through $\chi^{2}$ distribution with $\nu=m-1$ degrees of freedom. The hypotheses are

$$
\begin{aligned}
& H_{0}: \bar{\sigma}=\hat{\sigma} \\
& H_{1}: \bar{\sigma} \neq \hat{\sigma}
\end{aligned}
$$

The null hypothesis cannot be rejected when the value of test statistic $H$ lies in the interval $\left[\chi_{\alpha / 2, \nu}^{2}, \chi_{1-\alpha / 2, \nu}^{2}\right]$, where $1-\alpha$ is confidence level.

In the next section we are first going to represent the results of proposed algorithm and then the findings of evaluation methods results.

\section{RESULTS AND DISCUSSION}

\subsection{Results of proposed algorithm}

The entire procedure of target center acquisition is represented on the example of particular target "T203". The result of the first step - oblique plane adjustment is presented in Figure 2.

The precisions of the vectors in Figure 2 are in Table 1. All values are in millimeters 


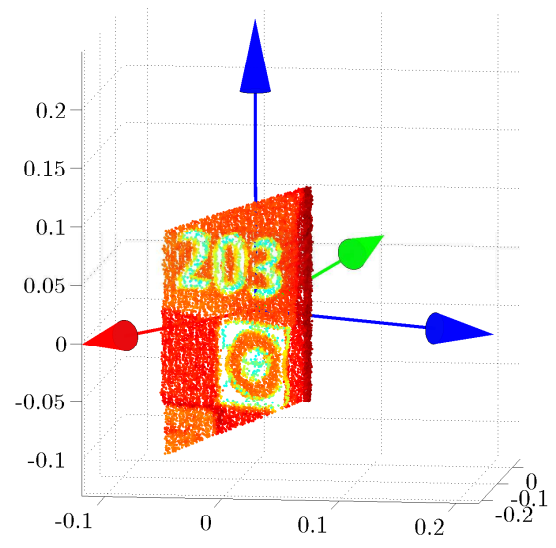

Figure 2: Raw point cloud colored by intensities, red vector is plane normal $\vec{n}$, blue vectors $\left(\overrightarrow{e_{1}}, \overrightarrow{e_{2}}\right)$ strain the plane and green vector is the direction of incidence laser beams $\vec{v}$

\begin{tabular}{|c|c|c|c|}
\hline & $\sigma_{x}$ & $\sigma_{y}$ & $\sigma_{z}$ \\
\hline$\vec{n}$ & 0.009 & 0.014 & 0.005 \\
$\overrightarrow{e_{1}}$ & 0.014 & 0.009 & 0.000 \\
$\overrightarrow{e_{2}}$ & 0.002 & 0.015 & 0.015 \\
\hline
\end{tabular}

Table 1: Precisions of unit vectors defining the reference plane

The precision of parameter $d$ from equation of a plane (1) equals $\sigma_{d}=0.06 \mathrm{~mm}$. The incidence angle of laser beams on to a reference plane equals $\varphi=66.3^{\circ}$.

In the Figure 3 points projected on to the plane and the obtained raster image are presented. Note that scan lines are visible due to oblique projection. If orthogonal projection was performed the scan lines would be blurred, if visible at all.

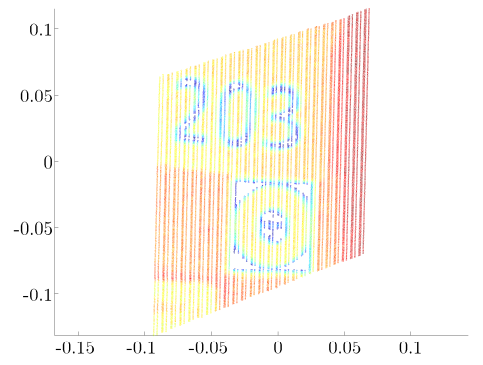

(a) Points projected to the plane

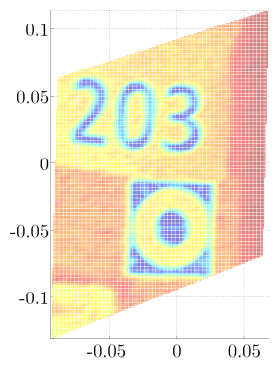

(b) Raster image

Figure 3: Points projected on to the reference plane

Image matching was performed next:

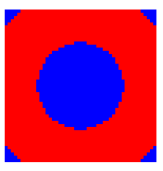

(a) Template

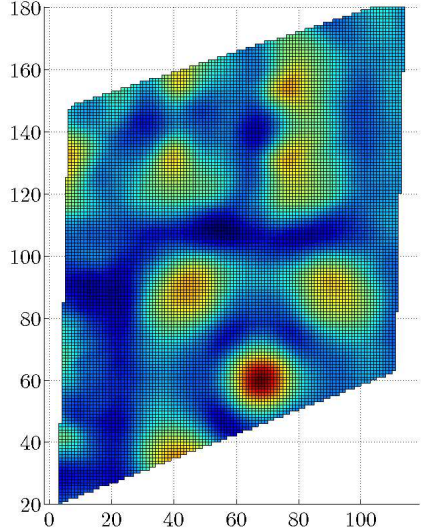

(b) Correlation coeficients

Figure 4: Template and correlations of image matching

The greatest correlation coefficient equals $\rho_{\max }=0.88$

The results of image matching are image coordinates $x_{i}$, $y_{i}$ with precisions

$\sigma_{x i}=0.03749$

$\sigma_{y i}=0.03745$

Image coordinates have to be transformed into plane coordinates (coordinate system defined by $\mathbf{e}_{1}$ and $\mathbf{e}_{2}$ in meters). They need to be multiplied by the size of the pixel expressed in meters and added to the position of the corner of the image in the plane coordinate system. Precisions of coordinates $x_{c}, y_{c}$ and $z_{c}$ are

$$
\begin{aligned}
& \sigma_{x c}=0.03749 \mathrm{~mm} \\
& \sigma_{y c}=0.03745 \mathrm{~mm} \\
& \sigma_{z c}=0.00006 \mathrm{~mm}=\sigma_{d}
\end{aligned}
$$

In the end, the target center is transformed from the plane back into the original point cloud, where all precision must be taken into account (see eqn. (7),(8),(9)). The accuracies of target center coordinates are

$$
\begin{aligned}
\sigma_{X} & =0.046 \mathrm{~mm} \\
\sigma_{Y} & =0.095 \mathrm{~mm} \\
\sigma_{Z} & =0.085 \mathrm{~mm}
\end{aligned}
$$

The reference plane best fits all the points of the point cloud. We wished to find the plane that only fit the points belonging to the target. The original point cloud is cropped around the obtained center, with the radius of the target $(r=4 \mathrm{~cm})$. The process is than repeated on the cropped point cloud and the results are

$$
\begin{aligned}
\sigma_{X} & =0.060 \mathrm{~mm} \\
\sigma_{Y} & =0.094 \mathrm{~mm} \\
\sigma_{Z} & =0.036 \mathrm{~mm}
\end{aligned}
$$

\subsection{Correctness of the results}

An incorrectly determined target plane, due to great incidence angles, gross errors or data artefacts (Lichti and Skaloud, 2010b) in the scanning process, or obstacles between the scanner and target are the main reasons for wrong results. As long as the target is at least roughly visible in the raster image, it is very likely that image matching will extract its center correctly. This property of the algorithm allows us to carry out visual analysis of 
correctness of our method. It would be best if the algorithm was able to automatically detect erroneous results. The decision may be based on the values of incidence angle and maximal correlation between the raster image and the template.

The 65 targets distributed over the walls and ceiling of a room were scanned from four scanner stations with Riegl VZ-400 and Leica C10 TLS. Some technical characteristic of scanners are given in Table 2.

\begin{tabular}{|c|c|}
\hline Riegl - VZ400 & Leica C10 \\
\hline \hline \multicolumn{2}{|c|}{ Single Point Precision } \\
\hline \multicolumn{2}{|c|}{ Laser Beam Divergence } \\
$\begin{array}{c}\sigma_{\text {distance }}=5 \mathrm{~mm} \\
\text { angle resolution } 1.8^{\prime \prime}\end{array}$ & $\begin{array}{c}\sigma_{\text {position }}=6 \mathrm{~mm} \\
\sigma_{\text {angle }}=12^{\prime \prime}\end{array}$ \\
\hline \multicolumn{2}{|c|}{ Angular Step } \\
\hline $3.5 \mathrm{~mm} / 10 \mathrm{~m} \sim 72^{\prime \prime}$ & $7 \mathrm{~mm}$ (at $1-50 \mathrm{~m})$ \\
\hline $8.5^{\prime \prime}$ & $<1 \mathrm{~mm} /$ full range $\sim 4^{\prime \prime}$ \\
\hline
\end{tabular}

Table 2: Technical specification of used scanners

The results of the correctness analysis are shown in the Figure 5. Brown and blue dots represent correctly and incorrectly determined target center, respectively. Green dots represent special cases where higher reflectances were measured on the black surface. In such cases an inverse template (switched zeros and ones) achieves correct result.

When scanning with Riegl we decided not to scan targets at incidence angles greater than $80^{\circ}$, while with Leica we scanned all targets. The general conclusion based on Figure 5 can be: when the incidence angle is lower than $80^{\circ}$ and the maximal correlation in image matching is greater than 0.6 the target should be determined correctly. However, even some targets with lower correlation may be determined corretly but not for incidence angles lower than $10^{\circ}$ or greater than $80^{\circ}$. We suggest that targets with correlation between 0.4 and 0.6 are verified manually. When the correlation is lower than 0.6 and the incidence angle lower than $5^{\circ}$, one should repeat image matching with an inverse template (switched ones and zeros).

\subsection{Results of statistical testing}

The comparison of variances (standard deviations) described in Section 3 returned the results shown in Table 3. All values of standard deviations are in millimeters.

\begin{tabular}{|c|c|c|c|c|c|c|c|c|}
\hline & $\varphi\left[{ }^{\circ}\right]$ & $d[\mathrm{~m}]$ & $\hat{\sigma_{x}}$ & $\hat{\sigma_{y}}$ & $\hat{\sigma_{z}}$ & $\overline{\sigma_{x}}$ & $\overline{\sigma_{y}}$ & $\overline{\sigma_{z}}$ \\
\hline \hline T1 & 2 & 4.2 & 0.20 & 0.32 & 0.07 & 0.04 & 0.03 & 0.04 \\
\hline T2 & 15 & 4.2 & 0.22 & 0.39 & 0.13 & 0.03 & 0.04 & 0.03 \\
\hline T3 & 35 & 4.2 & 0.37 & 0.52 & 0.33 & 0.11 & 0.14 & 0.17 \\
\hline T4 & 50 & 4.3 & 0.25 & 0.39 & 0.16 & 0.04 & 0.05 & 0.04 \\
\hline T5 & 80 & 4.3 & 0.54 & 2.52 & 1.66 & 1.08 & 0.18 & 0.27 \\
\hline T6 & 10 & 45.8 & 0.10 & 0.18 & 0.10 & 0.02 & 0.02 & 0.02 \\
\hline T7 & 65 & 45.9 & 0.41 & 0.37 & 0.22 & 0.06 & 0.04 & 0.03 \\
\hline
\end{tabular}

Table 3: Empirical and theoretical standard deviations

For $\nu=59$ degrees of freedom and confidence level of 99 $\%$, the confidence interval is $[34.77,90.71]$. The obtained test statistic for all coordinate components of all targets are in Table 4.

Red values mean that theoretical precision (estimated through the procedure) is statistically significantly overestimated (values are to low). Blue values mean that it is

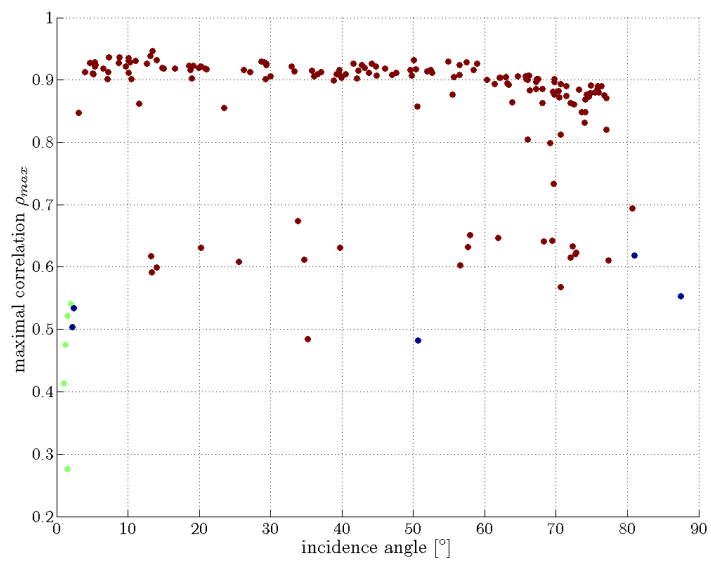

(a) Riegl - VZ400

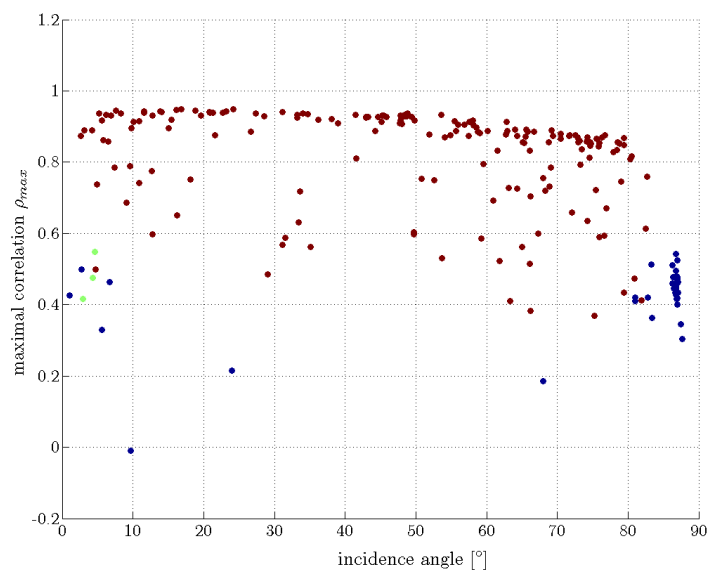

(b) Leica C10

Figure 5: Correctness of results

statistically significantly underestimated. The third option would mean (if it appeared) that it is not possible to claim that theoretical precision is different from empirical. All three statements are stated with the confidence level of $99 \%$.

The results of statistical testing suggest that the process of precision estimation produces too optimistic results. If we check the orientation of coordinate system axes we find that the main reason is a too optimistically determined value of parameter $d$ of the projection plane, which corresponds to the dispersion of points around the plane. The vast number of points forces the precision of their mean to be unreasonably high.

However, despite of the negative results of statistical testing we must not overlook the actual empirical precisions of the results. The majority of estimated precisions stay

\begin{tabular}{|c|c|c|c|}
\hline target & $H_{x}$ & $H_{y}$ & $H_{z}$ \\
\hline \hline T1 & 2.30 & 0.51 & 20.19 \\
\hline T2 & 0.92 & 0.50 & 2.78 \\
\hline T3 & 5.64 & 4.12 & 16.58 \\
\hline T4 & 1.75 & 0.95 & 3.02 \\
\hline T5 & 234.16 & 0.31 & 1.56 \\
\hline T6 & 1.33 & 0.59 & 2.43 \\
\hline T7 & 1.10 & 0.76 & 1.05 \\
\hline
\end{tabular}

Table 4: values of $\mathrm{H}$ test statistic 
significantly below half millimeter which seems to be an encouraging result. The target "T5" must be excluded from the former state, confirming the findings of previous experiment that the results are incorrect at incidence angles greater than $80^{\circ}$. Detailed investigation reveals that in most cases the dispersion of measured ranges $\rho$ was too high to allow proper plane adjustment.

\section{CONCLUSIONS AND FUTURE WORK}

The majority of today's TLS software has integrated algorithms for planar target center determination. The drawback of such programs is an unknown algorithm and only a flat rate estimation of center precision.

The proposed algorithm allows us to determine the center of a planar target with precisions significantly higher than the scanning resolution.

Different types and sizes of targets can be processed, and only the template image has to be changed. An important advantage is that targets can be homemade, printed on normal paper.

The fact that target centers can be determined properly even when scanned under an incidence angle of up to $80^{\circ}$, allows us to use planar targets for tasks where spherical targets are usually used.

In this paper we present the details of a proposed algorithm with emphasis on the precision estimation of every step. In the practical example the results of every step are presented numerically and graphically. At the end of the process we obtain target center coordinates along with its precisions. At first glance precisions seem to be severely overestimated. Further investigation with statistical testing confirmed that theoretical precisions are indeed to high but empirical precisions were still about half millimeter.

Another test revealed that it is possible to automatize the procedure for the detection of probably incorrect results.

With further investigations we would like to improve the error propagation procedure in a way that theoretical precision will get in accordance with empirical ones. More tests with different sizes of targets, different scanning resolutions, and at different ranges have to be carried out to confirm the applicability of the algorithm.

When scanning highly contrasting shapes it is common to obtain different ranges on dark and light surface. It may also be the property of material the target is made of. The target has to be scanned at a known distance from the scanner to find which color of the surface ensures accurate distance measurements. When it is known, the plane can be adjusted only through the points belonging to the right color.

In this paper we focused mainly on precisions while accuracies were set aside. Targets can be designed in a way to allow the centers to be measured with theodolite. This way we can assure correct target center positions and compare it to centers acquired by TLS. A test requires a lot of caution not to attach scanners' systematic errors to the target acquisition algorithm.

In conclusion we would like to point out the most important emphasis of this paper. Oblique plane adjustment and oblique projection of points on to the plane are important to ensure accurate results. The proposed algorithm evaluates the precision of result which is empirically proven within the range of half millimeter. Target centers can be properly determined even if scanned at an incidence angle of up to $80^{\circ}$.

\section{ACKNOWLEDGEMENTS}

The authors would like to thank the DFG consulting d.o.o. and Piniped d.o.o. for rental of laser scanners.

\section{REFERENCES}

Chow, J. C. K., Teskey, W. F. and Lovse, J. W. B., 2011. Insitu Self-calibration of Terrestrial Laser Scanners and Deformation Analysis Using Both Signalized Targets and Intersection of Planes for Indoor Applications. In: 14th FIG Symposium on Deformation Measurements and Analysis, 5th IAG Symposium on Geodesy for Geotechnical and Structural Engineering, and 2nd International Workshop on Spatial Information Technologies for Monitoring the Deformation of Large-Scale Man-Made Linear Features, Hong Kong, China, p. 15.

Chow, J., Ebeling, A. and Bill, T., 2010. Low cost Artificial Planar Target Measurement Techniques for TLS. In: FIG Congress 2010 Facing the Challenges - Building the Capacity, Sydney, Australia, p. 13.

Fischler, M. A. and Bolles, R. C., 1981. Paradigm for Model model fitting with applications to image analysis and automated cartography. Graphics and Image Processing 24(6), pp. 381395 .

Grigillo, D. and Stopar, B., 2003. Methods of gross error detection in geodetic observations. Geodetski Vestnik 47(4), pp. 387403. (in Slovene).

Höhle, J., 1997. The Automatic Measurement of Targets. Photogrammetrie Fernerkundung Geoinformation (1), pp. 13-21.

Kraus, K., 2000. Photogrammetry. 2nd englis edn, Walter De Gruyter.

Kuang, S., 1996. Geodetic Network Analysis and Optimal design. Ann Arbor Press, Inc., Chelsea, Michigan.

Lichti, D. D., 2010. A review of geometric models and selfcalibration methods for terrestrial laser scanners. Boletim De Ciencias Geodesicas 16, pp. 3-19.

Lichti, D. D. and Skaloud, J., 2010a. Airborne and terrestrial laser scanning. Whittles Publishing; CRC Press, chapter 3.3.1.1 Target-based regstration, pp. XVII, 318.

Lichti, D. D. and Skaloud, J., 2010b. Airborne and terrestrial laser scanning. Whittles Publishing; CRC Press, chapter 3.2.1.4 Data artefacts, pp. XVII, 318.

Lichti, D. D., Stewart, M. P., Tsakiri, M. and Snow, A. J. 2000. Calibration and testing of a terrestrial laser scanner. International Archives of Photogrammetry and Remote Sensing XXXIII(Part B5), pp. 485-492.

Reshetyuk, Y., 2010. A unified approach to self-calibration of terrestrial laser scanners. ISPRS Journal of Photogrammetry and Remote Sensing 65(5), pp. 445-456.

Schulz, T., 2007. Calibration of a Terrestrial Laser Scanner for Engineering Geodesy. PhD thesis, ETH Zürich, Zurich.

Schulz, T. and Ingensand, H., 2004. Influencing variables, precision and accuracy of terrestrial laser scanners. In: INGEO 2004 and FIG Regional Central and Eastern European Conference on Engineering Surveying, Bratislava, Slovakia, p. 9

Vezočnik, R., 2011. Analysis of terrestrial laser scanning technology for structural deformation monitoring. PhD thesis, University of Ljubljana. 